Published in final edited form as:

Clin Cancer Res. 2019 June 01; 25(11): 3374-3383. doi:10.1158/1078-0432.CCR-18-3049.

\title{
The dual PI3K/mTOR-pathway inhibitor GDC-0084 achieves antitumor activity in PIK3CA-mutant breast cancer brain metastases
}

\author{
Franziska M. Ippen ${ }^{1,2}$, Christopher A. Alvarez-Breckenridge ${ }^{3}$, Benjamin M. Kuter ${ }^{1}$, \\ Alexandria L. Fink ${ }^{3}$, Ivanna V. Bihun ${ }^{1}$, Matthew Lastrapes ${ }^{4}$, Tristan Penson ${ }^{3}$, Stephen P. \\ Schmidt ${ }^{5}$, Gregory R. Wojtkiewicz ${ }^{6}$, Jianfang Ning ${ }^{3}$, Megha Subramanian ${ }^{1}$, Anita Giobbie- \\ Hurder $^{7}$, Maria Martinez-Lage ${ }^{8}$, Scott L. Carter ${ }^{4}$, Daniel P. Cahill ${ }^{3}$, Hiroaki Wakimoto ${ }^{3}$, \\ Priscilla K. Brastianos ${ }^{1}$ \\ ${ }^{1}$ Cancer Center, Massachusetts General Hospital, Harvard Medical School, Boston, MA \\ ${ }^{2}$ Department of Neurology, Heidelberg University Hospital, Heidelberg, Germany \\ ${ }^{3}$ Department of Neurosurgery, Massachusetts General Hospital, Harvard Medical School, Boston, \\ MA \\ 4Joint Center for Cancer Precision Medicine, Dana-Farber Cancer Institute/Brigham and Women's \\ Hospital, Harvard Medical School, Boston, MA \\ ${ }^{5}$ Center for Systems Biology, Massachusetts General Hospital, Harvard Medical School, Boston, \\ MA \\ ${ }^{6}$ Department of Radiology, Massachusetts General Hospital, Harvard Medical School, Boston, \\ MA \\ ${ }^{7}$ Department of Biostatistics \& Computational Biology, Dana-Farber Cancer Institute, Boston, MA \\ ${ }^{8}$ Department of Pathology, Massachusetts General Hospital, Harvard Medical School, Boston, MA
}

\section{Abstract}

Corresponding author: Priscilla K. Brastianos, MD, Cancer Center, Massachusetts General Hospital, 55 Fruit Street, Boston, MA, 02114, PBRASTIANOS@mgh.harvard.edu, Phone number: 617-724-1074.

Authors' Contributions

Conception and design: F.M. Ippen, D.P. Cahill, H. Wakimoto, P.K. Brastianos

Development of methodology: F.M. Ippen, H. Wakimoto, P.K. Brastianos

Acquisition of data: F.M. Ippen, C.A. Alvarez-Breckenridge, B.M. Kuter, A.L. Fink, I.V. Bihun, T. Penson, S.P. Schmidt, G.R.

Wojtkiewicz, J. Ning

Analysis and interpretation of data: F.M. Ippen, A. Giobbie-Hurder, M. Lastrapes, S.P. Schmidt, G.R. Wojtkiewicz, Scott L. Carter,

H. Wakimoto, P.K. Brastianos

Writing, review, and/or revision of the manuscript: F.M. Ippen, M. Subramanian, D.P. Cahill, H. Wakimoto, P.K. Brastianos

Administrative, technical, or material support: F.M. Ippen, P.K. Brastianos

Study supervision: D.P. Cahill, H. Wakimoto, P.K. Brastianos

Other (interpreted the histological analysis): Maria Martinez-Lage Alvarez

Disclosure of Potential Conflicts of Interest

F.M. Ippen is supported by the Deutsche Forschungsgemeinschaft (DFG, German Research Foundation- Projektnummer: 325246018).

P.K. Brastianos received honoraria from Genentech and Merck, consultant fees from Lilly, Tesaro, Genentech-Roche and Angiochem and research funding to MGH from Merck and Pfizer. D.P. Cahill received honoraria from Lilly. The other authors declare no potential conflicts of interest. 
Purpose: Previous studies have shown that the PI3K/Akt/mTOR-pathway is activated in up to $70 \%$ of breast cancer brain metastases, but there are no approved agents for affected patients. GDC-0084 is a brain penetrant, dual PI3K/mTOR-inhibitor that has shown promising activity in a preclinical model of glioblastoma. The aim of this study was to analyze the efficacy of PI3K/ mTOR blockade in breast cancer brain metastases models.

Experimental methods: The efficacy of GDC-0084 was evaluated in PIK3CA-mutant and PIK3CA-wildtype breast cancer cell lines and the isogenic pairs of PIK3CA-wildtype and -mutant (H1047R/+) MCF10A cells in vitro. In vitro studies included cell viability and apoptosis assays, cell cycle analysis and Western blots. In vivo, the effect of GDC-0084 was investigated in breast cancer brain metastasis xenograft mouse models and assessed by bioluminescent imaging and immunohistochemistry.

Results: In vitro, GDC-0084 considerably decreased cell viability, induced apoptosis and inhibited phosphorylation of Akt and p70 S6 kinase in a dose-dependent manner in PIK3CAmutant breast cancer brain metastatic cell lines. In contrast, GDC-0084 led only to growth inhibition in PIK3CA-wildtype cell lines in vitro. In vivo, treatment with GDC-0084 markedly inhibited the growth of PIK3CA-mutant, with accompanying signaling changes, and not PIK3CAwildtype brain tumors.

Conclusion: The results of this study suggest that the brain-penetrant PI3K/mTOR-targeting GDC-0084 is a promising treatment option for breast cancer brain metastases with dysregulated PI3K/mTOR signaling pathway conferred by activating PIK3CA mutations. A national clinical trial is planned to further investigate the role of this compound in patients with brain metastases.

\section{Keywords}

brain metastases; breast cancer; PI3K-inhibition; GDC-0084

\section{Introduction}

Brain metastases are the most common intracranial neoplasm in adult cancer patients and are associated with significant morbidity and mortality $(1,2)$. Advances in systemic therapies and neuroimaging modalities, earlier tumor detection, and longer survival of cancer patients have contributed to an increased incidence of brain metastases $(2,3)$. Despite the use of multidisciplinary treatment approaches including surgery, stereotactic radiosurgery (SRS) and/or whole-brain radiation therapy (WBRT), the median survival of affected patients still remains poor, ranging from 4-18 months $(4,5)$. As breast cancer accounts for the most common malignancy in women worldwide (6) and the second most frequent primary tumor causing brain metastases (7), the management of affected patients is a growing challenge.

Recent studies have shown that activation of the phosphatidylinositol 3-kinase (PI3K) pathway occurs in up to $70 \%$ of patients with breast cancer brain metastases (BCBM) (810). Oncogenic alterations in phosphatidylinositol-4,5-bisphosphate 3-kinase catalytic subunit alpha (PIK3CA) lead to enhanced activation of the PI3K/protein kinase B (Akt)/ mammalian target of rapamycin (mTOR) pathway, which has been shown to promote the development, progression, and treatment resistance of various cancer types, including HER2-positive breast cancer(11). Inhibition of this crucial pathway presents an appealing 
strategy for the treatment of BCBM. Although numerous inhibitors targeting this pathway have been developed in recent years, there are no FDA-approved PI3K inhibitors for the treatment of brain metastases $(12,13)$. PI3K/Akt/mTOR-pathway inhibitors that have been investigated to date have either shown only modest uptake in the brain (14) or have mainly been tested for their efficacy in the context of primary brain tumors (15-19), breast cancer (20) and brain metastases arising from other primary histologies (21-23).

The blood-brain barrier (BBB) poses an additional challenge for drug administration in the central nervous system (CNS), as treatment of brain metastases necessitates compounds which are optimized to cross this biologic barrier $(22,24)$. A PI3K-inhibitor that can effectively penetrate the BBB and achieve metabolic stability within the brain presents a promising approach for the treatment of patients with PIK3CA-mutant BCBM. GDC-0084 is a dual $\mathrm{PI} 3 \mathrm{~K} / \mathrm{mTOR}$ inhibitor that is specifically optimized to cross the $\mathrm{BBB}$, achieve good metabolic stability within the brain, has low efflux ratios (25) and has recently been shown to achieve significant tumor growth inhibition in preclinical models of glioblastoma and cutaneous squamous cell carcinoma (cSCC) $(26,27)$. The role of GDC-0084 in brain metastases has not been investigated. To that end, our aim was to investigate the efficacy of this inhibitor in PIK3CA-mutant (MT) and -wildtype (WT) BCBM in vitro and in vivo.

\section{Methods}

\section{Cell lines}

We used human metastatic breast cancer cell lines that were PIK3CA-mutant (JIMT-1 BR-3 (PIK3CA C420R) (28); MDA-MB-361 (PIK3CA E545K) (29)) and PIK3CA-wildtype (MDA-MB-231 BrM2, BS-004). The isogenic non-tumorigenic epithelial breast cell lines MCF10A (parental, PIK3CA-WT) and PIK3CA (H1047R/+) MCF10A (heterozygous knockin of PIK3CA-kinase domain activating mutation, PIK3CA-MT) were also used in in vitro validation experiments. The human epidermal growth factor receptor (HER2) positive and estrogen and progesterone receptor (ER/PR) negative cell line JIMT-1 BR-3 (HER2+, ER/PR-) was kindly provided by the laboratory of Dr. Patricia Steeg (National Cancer Institute, Bethesda, Maryland) and the cell line MDA-MB-231 BrM2 (HER2-, ER/PR-) was a generous gift from the laboratory of Dr. Joan Massagué (Memorial Sloan Kettering Cancer Center, New York City, New York). MDA-MB-361 (HER2+, ER/PR+) was purchased from ATCC. BS-004 (HER2+, ER/PR+) was derived from a patient's excised breast cancer brain metastasis. The patient provided written consent. The study was reviewed and approved by the human subjects institutional review board (IRB) of the Dana-Farber Cancer/Harvard Cancer Center, and the research performed in accordance with the Declaration of Helsinki. The epithelial breast cell lines MCF10A (HER2-, ER/PR-) and PIK3CA (H1047R/+) MCF10A (HER2-, ER/PR-) were purchased from Horizon Discovery. JIMT-1 BR-3, MDAMB-231 BrM2 and BS-004 cells were cultured in Dulbecco's modified Eagle medium (DMEM) supplemented with 10\% fetal bovine serum (FBS) and 1\% penicillinstreptomycin-amphotericin B. MDA-MB-361 was cultured in L15 supplemented with 20\% fetal bovine serum (FBS) and 1\% penicillin-streptomycin-amphotericin $\mathrm{B}$. The isogenic pairs MCF10A and PIK3CA (H1047R/+) MCF10A were cultured in DMEM/F-12 including $2.5 \mathrm{mM}$ L-glutamine and $15 \mathrm{mM}$ HEPES, supplemented with $5 \%$ horse serum, $10 \mu \mathrm{g} / \mathrm{mL}$ 
insulin, $0.5 \mu \mathrm{g} / \mathrm{mL}$ hydrocortisone, $0.1 \mu \mathrm{g} / \mathrm{mL}$ cholera toxin and $0.2 \mathrm{ng} / \mathrm{mL}$ EGF (30). The PIK3CA mutation status of all cancer cell lines was confirmed via whole-exome sequencing.

All cell lines were confirmed to be mycoplasma-free and were tested throughout the course of the experiments every month (PCR Mycoplasma Detection Kit, abm).

\section{Viral vectors and transduction of cell lines}

Cell lines were engineered to express Firefly luciferase and mCherry $(\mathrm{FmC})$ by transduction with the lentiviral construct LV-pico2-Fluc-mCherry (pLV-FmC), which was kindly provided by Khalid Shah (Brigham and Women's Hospital; Boston, MA)/Dr. Andrew Kung (Dana Farber Cancer Institute; Boston, MA). JIMT-1 BR-3 and MDA-MB-231 BrM2 cells were transduced at a multiplicity of infection (MOI) of 2 in media containing Polybrene (8 $\mu \mathrm{g} / \mathrm{mL}$; EMD Millipore; Burlington, MA) for 48 hours. Cells were selected with puromycin ( $7 \mu \mathrm{g} / \mathrm{mL}$ ) for 3 days, visualized by fluorescence microscopy for mCherry to confirm successful transduction, and then sorted for mCherry expression using fluorescenceactivated cell sorting (FACSAria Cell-Sorting System, BD Biosciences).

\section{PI3K/Akt/mTOR-pathway inhibitor}

To investigate blockade of the PI3K/Akt/mTOR-pathway, the dual PI3K/mTOR-inhibitor GDC-0084 was used. GDC-0084 was kindly provided by Genentech. The inhibitor was added at concentrations ranging from $0.25 \mu \mathrm{mol} / 1$ up to $10 \mu \mathrm{mol} / 1$ to cell culture medium with cells plated at 50-70\% confluency in in vitro studies. Controls were incubated with $0.1 \%$ DMSO. The dose of GDC-0084 was chosen based on other preclinical studies in brain tumors with PI3K/Akt/mTOR-inhibitors $(21,31)$. GDC-0084 was diluted in DMSO for in vitro studies and in a combination of $0.5 \%$ methylcellulose and $0.2 \%$ Tween 80 for in vivo studies.

\section{Cell viability and apoptosis assays}

Cells were plated in triplicates for cell viability assays and in quadruplicates for apoptosis assays at a density of 5000 cells per well on a 96-well plate. Cell lines were treated with GDC-0084 the next day in concentrations ranging from $0.25 \mu \mathrm{mol} / 1$ to $10 \mu \mathrm{mol} / 1$ for 10 hours (apoptosis assays) and 72 hours (cell viability assays). Controls were incubated with $0.1 \%$ DMSO. After treatment, cells were lysed using the Caspase-Glo 3/7 (Promega, apoptosis assays) or the CellTiter-Glo (Promega, cell viability assays) reagent. The luminescence generated by these reagents is proportional to the Caspase 3/7-activity or the amount of viable cells respectively, and was read using a Synergy HT multi-detection microplate reader (BioTek). The percentage of Caspase 3/7 or viable cells was calculated relative to DMSOincubated controls.

\section{Cell cycle analysis}

Cells were plated in triplicates at a density of $3.5 \times 10^{5}$ in $60 \mathrm{~mm}$ well plates and treated with GDC-0084 (control, $1 \mu \mathrm{M}, 2.5 \mu \mathrm{M}, 5 \mu \mathrm{M}, 7.5 \mu \mathrm{M}$ and $10 \mu \mathrm{M}$ ) the next day for a total of 72 hours. Afterwards, floating and adherent cells were harvested, washed with PBS and fixed in ice-cold 70\% ethanol for 24 hours. Subsequently, cells were washed with PBS again. Fixed cells were stained using the Propidium Iodide Flow Cytometry Kit (ab139418, abcam) with 
$200 \mu \mathrm{l}$ of propidium iodide (PI) staining solution $(500 \mu \mathrm{l} 20 \mathrm{X}$ propidium iodide and $50 \mu \mathrm{l}$ 200X RNAse in $9.45 \mathrm{ml} \mathrm{PBS}$ ) and incubated at $37^{\circ} \mathrm{C}$ in the dark for 30 minutes. The cell cycle analysis was conducted using the BD LSRII and BD FACSDiva Software Version: 8.0.1 (BD Biosciences) and analyzed using FlowJo software version 10 (LLC, Ashland Oregon).

\section{Western blots}

Cells were plated in triplicates in $60 \mathrm{~mm}$ well plates at a density of $3.5 \times 10^{5}$ cells and treated with GDC-0084 (control, $1 \mu \mathrm{M}, 2.5 \mu \mathrm{M}, 5 \mu \mathrm{M}, 7.5 \mu \mathrm{M}$ and $10 \mu \mathrm{M}$ ) the next day for a total of 6 hours. Afterwards, cells were harvested and lysed in radioimmunoprecipitation (RIPA) buffer (Thermo Fisher Scientific) containing protease and phosphatase inhibitor cocktails

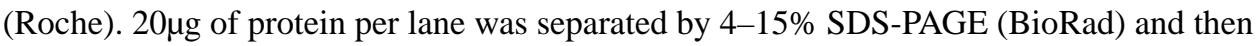
transferred to polyvinylidene difluoride membranes (BioRad) by electroblotting. Membranes were blocked with $5 \%$ nonfat dry milk in TBST (20 mM Tris pH7.5, $150 \mathrm{mM} \mathrm{NaCl}, 0.1 \%$ Tween20) for 1 hour at room temperature and then incubated with primary antibodies (pAkt (Ser473) \#4060, Akt \#9272, p-p70 S6 Kinase (Thr389) \#9205, p70 S6 Kinase \#2708, pp44/42 MAPK (Erk1/2) (Thr202/Tyr204) \#4370, p-MEK1/2 (Ser217/221) \#9154, p-p90RSK (Ser380) \#11989, p-MSK1 (Thr581) \#9595, $\beta$-Actin \#3700, all from Cell Signaling Technology) at $4^{\circ} \mathrm{C}$ overnight. Membranes were washed with TBST and incubated with horseradish peroxidase-conjugated secondary antibodies (anti-rabbit IgG $(\mathrm{H}+\mathrm{L}), \mathrm{HRP}$ conjugate and anti-mouse $\operatorname{IgG}(\mathrm{H}+\mathrm{L}), \mathrm{HRP}$ conjugate, both from Promega) for 1 hour at room temperature. Thereafter, membranes were washed again in TBST and signals were visualized with an ECL blotting substrate (Thermo Fisher Scientific). Blots were analyzed using Image Lab (BioRad) and cropped in Adobe illustrator software version CC 2018 (San José, CA).

\section{Animal studies}

All in vivo mouse experiments were approved by the Institutional Animal Care and Use Committee (IACUC) at Massachusetts General Hospital.

Stereotactic intracranial tumor implantation-A total of 32 animals were analyzed for imaging ( 8 animals per cohort) and a total of 4 animals in a separate cohort were analyzed for immunohistochemistry. Prior to surgery, 8 -week old female athymic nu/nu mice (Charles River Laboratories, Wilmington, MA) were anesthetized with pentobarbital (i.p. injection, $40-70 \mathrm{mg} / \mathrm{kg}$ ). Anesthetized mice were placed into a stereotactic apparatus and the head was stabilized by the ear bars. After opening the skin over the skull, Bregma was identified, and a total of $10 \times 10^{4}$ JIMT-1 BR-3 cells or $7.5 \times 10^{4}$ MDA-MB-231 BrM2 cells in $4 \mu$ l Hanks' Balanced Salt Solution (HBSS) were stereotactically implanted into the right striatum ( $2 \mathrm{~mm}$ lateral from Bregma, 2.5-mm deep) with a Hamilton syringe. We chose these two cell lines because they have similar growth patterns and doubling times.

Afterwards, the skull was sealed with bone wax and the wound was sutured. Postoperatively, all mice were provided carprofen (MediGel CPF, 2-oz cup, Clear $\mathrm{H}_{2} \mathrm{O}$, Portland, ME) for a total of 3 days. 
Bioluminescent imaging and analysis of tumor burden-Seven days after the surgical procedure, mice were imaged for the first time via bioluminescent imaging (BLI). Dynamic imaging was performed to assess their initial tumor burden. Before undergoing this imaging procedure, mice were anesthetized with isoflurane and injected with $4.5 \mathrm{mg}$ D-

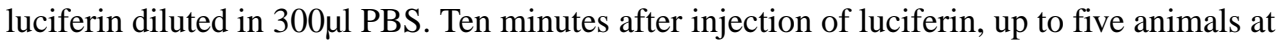
a time were imaged on the Spectral Ami HTX (Spectral Instruments Imaging, Tuscon, AZ). Luminescent exposure times of $60 \mathrm{~s}, 1 \mathrm{~s}$ and $0.5 \mathrm{~s}$ at $5 \mathrm{~min}$. intervals were chosen until the peak luminescent signal from the whole mouse body was reached. The tumor burden was analyzed by subtracting the background signal from the signal above the mouse's cranium and measured in total flux (p/s) with the software Aura version 2.2.1.0 (Spectral Instruments Imaging, Tuscon, AZ). Figures were generated with the software Amira version 5.3.2 (Thermo Fisher Scientific, Houston, TX) using the binning 2, 0.5s exposure and background subtracted image. To ensure accurate measurement of tumor burden throughout the entire study, mice received a baseline dynamic BLI scan 7 days post tumor inoculation. Mice harboring intracranial tumors with a similar tumor burden and a minimum flux of 10.000.000 p/s in JIMT-1 BR-3 tumors and of 400.000 p/s (flux) in MDA-MB-231 BrM2 tumors were selected for further experiments. Mice were subsequently randomized to GDC-0084 or sham treatment, which was initiated the day after (day 8 post implantation). BLI-based tumor burden on day 7 (pre-treatment) was not statistically different between the GDC-0084 treatment and sham groups. Afterwards, BLI was performed on a weekly basis until the end of the study at 35 days post-intracranial tumor implantation to monitor tumor growth in all mice, as measured by flux (p/s). Due to the large magnitude of the total flux $(\mathrm{p} / \mathrm{s})$ values, all data were $\log _{10}$-transformed prior to analysis. The primary endpoint of this study was the difference in tumor burden measured in total photon flux $(\mathrm{p} / \mathrm{s})$ between the cohorts that received GDC-0084 vs. sham over a period of 35 days. This period was chosen in order to avoid reductions in BLI-signals due to necrosis and therefore, an underestimation of the actual tumor burden as a result (32).

Treatment and monitoring-The dual PI3K/mTOR-inhibitor GDC-0084 was administered daily via oral gavage $(15 \mathrm{mg} / \mathrm{kg})$ for a total of 28 days. Mice randomized to treatment received $15 \mathrm{mg} / \mathrm{kg}$ GDC-0084 diluted in $0.5 \%$ methylcellulose and $0.2 \%$ Tween 80 per day, mice randomized to sham received a weight-adapted dose of $0.5 \%$ methylcellulose and $0.2 \%$ Tween 80 daily. Mice were monitored daily following stereotactic intracranial tumor injection and sacrificed at $\geq 20 \%$ weight loss, the onset of neurological symptoms or at the end of the study which was no later than 35 days post-implantation. In the separate mouse cohort that was analyzed for immunohistochemistry, brains were harvested 14 days after intracranial tumor cell implantation and after 7 daily dosings.

\section{Immunohistochemistry}

Mouse brains were fixed in $10 \%$ formalin for 24 hours and subsequently embedded in paraffin. For immunohistochemistry, $5 \mu \mathrm{m}$ thick sections were deparaffinized. Manual staining was conducted for pAkt and pS6 ribosomal protein. For pAkt and pS6 ribosomal protein staining, sections were treated with sodium citrate $(\mathrm{pH}=6)$ and heated for 10 minutes for antigen unmasking. Sections were blocked with TBST/5\% normal goat serum (NGS) and incubated in the following primary antibodies overnight: pAkt ((Ser473) \#4060, 1:50) and 
pS6 ribosomal protein ((Ser235/236) \#4858, 1:400, both from Cell Signaling Technology). The next day, sections were alternately washed in TBS and TBST and then incubated with the secondary antibody SignalStain Boost IHC Detection Reagent (HRP, Rabbit, \#8114, Cell Signaling Technology) for 30 minutes at room temperature. Afterwards, slides were stained with DAB (Dako) and counterstained with hematoxylin. Automated staining was conducted for p-p44/42 MAPK and pMEK1/2. Sections were treated with pre-diluted cell conditioning solution (CC2, Ventana), heated for 64 minutes and blocked in inhibitor CM (Ventana). Sections were incubated with the primary antibody p-p44/42 MAPK (Erk1/2) ((Thr202/ Tyr204) \#4370, 1:500) or p-MEK1/2 ((Ser221) \#2338, 1:50, both from Cell Signaling Technology) for 36 minutes and then with the secondary antibody OmniMap anti-Rb HRP (Multimer HRP, Ventana) for 12 minutes. Sections were then stained with DAB (Dako), counterstained with bluing reagent (Ventana) and post-counterstained with hematoxylin.

\section{Statistical analysis}

Statistical analysis was conducted with GraphPad Prism version 7 (GraphPad Software, La Jolla, CA) and SAS 9.4 (SAS Institute Inc., Cary, NC) for the mixed effects models. Graphs were cropped with the Adobe Illustrator software version CC 2018 (San José, CA). Evaluation of the efficacy of GDC-0084 in vivo was calculated by autoregressive linear mixed models. The outcome data of the treatment cohorts (GDC-0084 vs. sham), measured in total flux (p/s), were first $\log 10$-transformed. Differences between the treatment cohorts over time were assessed using linear mixed effects models, with an autoregressive covariance structure within mouse. Log10-transformed fold-changes in flux were the dependent variable in the mixed model. Independent predictors were treatment, time, and their interaction. A p-value of $<0.05$ was considered statistically significant in our analysis.

\section{Results}

\section{The dual PI3K/mTOR-inhibitor GDC-0084 inhibits cell proliferation and phosphorylation of Akt and p70 S6 kinase in PIK3CA-mutant breast cancer cell lines}

We used metastatic PIK3CA-mutant and PIK3CA-wildtype BCBM cell lines to investigate the anti-tumor efficacy of GDC-0084. We first performed in vitro assays to assess the survival of adherent PIK3CA-MT and PIK3CA-WT cell lines in response to increasing concentrations of GDC-0084.

GDC-0084 led to a considerable decrease in the proportion of viable cells (80-95\%) after 72 hours of treatment in PIK3CA-MT cell lines (JIMT-1 BR-3, MDA-MB-361), whereas a more modest decrease in viable cells $(40-60 \%)$ was observed in PIK3CA-WT cell lines (MDA-MB-231 BrM2, BS-004; Fig. 1A).

To determine whether this effect was mediated by apoptosis, Caspase-Glo 3/7 assays were conducted. The dual PI3K/mTOR-inhibitor robustly increased Caspase 3/7-activity in JIMT-1 BR-3 and MDA-MB-361 cells after 10 hours of treatment, but no such effect was seen in the MDA-MB-231 BrM2 and BS-004 cell lines (Fig. 1B).

Consistent with these findings, GDC-0084 also strongly inhibited phosphorylation of Akt and p70 S6 kinase in a dose-dependent manner in both PIK3CA-MT cell lines over a 
treatment course of 6 hours (Figure 1C). In contrast, no significant changes in phosphorylation of Akt and p70 S6 kinase were observed in response to increasing concentrations of GDC-0084 in the PIK3CA-WT cell lines over the same treatment duration.

We next used isogenic PIK3CA-MT and PIK3CA-WT epithelial breast cell lines to determine whether activating mutation of PIK3CA confers sensitivity to GDC-0084. GDC-0084 substantially decreased the proportion of viable cells (90-95\%) in PIK3CA-MT (H1047R/+) MCF10A cells compared to the PIK3CA-WT MCF10A cells (60-65\%) after 72 hours of treatment (Fig. 2A). Correspondingly, GDC-0084 greatly increased Caspase 3/7activity in the PIK3CA-MT (H1047R/+) MCF10A cell line, whereas a weaker effect was seen in the PIK3CA-WT parental cell line MCF10A (Fig. 2B). The highest apoptotic signal was obtained after 24 hours of treatment.

Previous studies have demonstrated that inhibition of components of the PI3K/Akt/mTORpathway can lead to compensatory upregulation of the Ras/Raf/MAPK signaling cascade in cancer cells $(33,34)$. In order to test whether dual PI3K/mTOR-pathway might affect activation of the MAPK-pathway in the context of BCBM, we assessed phosphorylation of p44/42 MAPK (Erk1/2), MEK1/2, p90RSK, and MSK1 by Western blots. We did not observe any significant differences in expression levels of p-p44/42 MAPK (Erk1/2), pMEK1/2, p-p90RSK, and p-MSK1 between PIK3CA-MT (Supplementary Fig. 1A) and WT BCBM cell lines (Supplementary Fig. 1B). Additionally, there was also no detectable change in expression of p-p44/42 MAPK (Erk1/2), p-MEK1/2, p-p90RSK, and p-MSK1 in response to treatment with GDC-0084 compared to sham in PIK3CA-MT and -WT cells.

Thus, GDC-0084 selectively inhibited the PI3K/Akt/mTOR-pathway and induced apoptotic cell death in PIK3CA-MT cell lines.

\section{GDC-0084 induces apoptosis in PIK3CA-mutant breast cancer cells but not PIK3CA- wildtype breast cancer cells}

To further investigate the reduction in cell viability in PIK3CA-MT cell lines, cell cycle analysis with PI-staining was conducted on all BCBM cell lines. In both cell lines harboring PIK3CA mutations, treatment with GDC-0084 for 72 hours yielded a dose-dependent increase in the sub-G1-phase (apoptotic cells) to approximately 70\% in JIMT-1 BR-3 (Fig. 3A and C) and 80\% in MDA-MB-361 (Fig. 3C and Supplementary Fig. 2A). For MDAMB-231 BrM2 (Fig. 3B and C), a mild trend towards an increase in the G1-fraction was observed, indicative of growth inhibition. GDC-0084 did not induce noticeable changes in cell cycle phases in BS-004 (Fig. 3C and Supplementary Fig. 2B). This corroborates our in vitro findings that GDC-0084 selectively induces apoptosis in PIK3CA-MT cell lines.

\section{GDC-0084 significantly inhibits growth of PIK3CA-mutant tumors in a patient-derived xenograft brain metastasis model}

In order to investigate whether GDC-0084 can effectively inhibit tumor growth in the brain in vivo, we orthotopically implanted either a PIK3CA-MT, trastuzumab-resistant (35) cell line (JIMT-1 BR-3) or a control PIK3CA-WT cell line (MDA-MB-231 BrM2) into the right striatum of female nude mice. 
Over the course of the treatment period (28 days), GDC-0084 was well tolerated and no adverse toxicities were observed. In the PIK3CA-MT JIMT-1 BR-3 model, BLI of tumor volume seven days after the initiation of treatment demonstrated GDC-0084-mediated growth inhibition of tumors, while sham-treated tumors rapidly grew (Fig. 4A). Throughout the duration of the study, GDC-0084 achieved highly significant inhibition in JIMT-1 BR-3 intracranial tumors (mixed effect model, $\mathrm{p}=0.0004$ for effect of treatment; $\mathrm{p}=0.0005$ for effect of time).

In contrast, no therapeutic benefit of GDC-0084 was noted in the PIK3CA-WT MDAMB-231 BrM2 xenograft model. Intracranial tumors continued to grow rapidly over time in both the sham and GDC-0084 cohorts (Fig. 4B). Accordingly, no significant differences between the two treatment cohorts were detected $(\mathrm{p}=0.80)$ and bioluminescent flux increased over time regardless of treatment $(\mathrm{p}<0.0001)$.

\section{GDC-0084 inhibits PI3K/Akt/mTOR signaling in PIK3CA-mutant breast cancer in the brain}

To assess whether GDC-0084 inhibited downstream molecular components of the PI3K/Akt/ mTOR-pathway in mouse brains, expression of pAkt and pS6 ribosomal protein was evaluated with immunohistochemistry. In PIK3CA-MT JIMT-1 BR-3 tumors, diminished immuno-staining of both pAkt (Ser473) and pS6 ribosomal protein (Ser235/236) was observed in the mouse brains treated with GDC-0084 as compared to those of the shamcohort (Fig. 5). This reduction in signal intensity was consistent with the phosphorylation inhibition seen in GDC-0084-treated PIK3CA-MT cell lines in vitro, and corroborates the observed tumor growth inhibition in vivo. In mice harboring PIK3CA-WT MDA-MB-231 BrM2 intracranial tumors, pAkt (Ser473) positivity was sparse, which was not altered by GDC-0084, and strong pS6 ribosomal protein was only slightly diminished by GDC-0084 treatment (Fig. 5). Collectively, systemic treatment with GDC-0084 potently inhibited PI3K/Akt/mTOR-pathway signaling and tumor growth in intracerebral xenografts generated with PIK3CA-MT breast cancer cells. In order to evaluate if dual PI3K/mTOR-blockade results in pro-survival activation of the Ras/Raf/MAPK-pathway as reported previously (33, 34), expression of p-p44/42 MAPK (Thr202/Tyr204) and pMEK1/2 (Ser221) was assessed with immunohistochemistry. In PIK3CA-MT JIMT-1 BR-3 intracranial tumors, pMEK1/2 was negative in both treatment and sham cohorts, and p-p44/42 MAPK was comparably positive in both cohorts (Supplementary Fig. 3). PIK3CA-WT MDA-MB-231 BrM2 brain tumors were negative for both p-p44/42 MAPK and pMEK1/2 regardless of treatment (Supplementary Fig. 3). In summary, we did not detect compensatory activation of the Ras/Raf/MAPK-pathway after treatment with GDC-0084 in vivo, in accordance with our in vitro results.

\section{Discussion}

Up to $50 \%$ of patients with HER2-positive breast cancer will develop brain metastases during their course of disease (36). To date, few effective targeted treatment options are available for patients with BCBM. Genomic studies of matched brain metastases and primary tumors $(9,10)$ as well as immunohistochemical analyses $(8)$, have shown that the $\mathrm{PI} 3 \mathrm{~K} / \mathrm{Akt} / \mathrm{mTOR}$-pathway is upregulated in up to $70 \%$ of brain metastases in affected breast 
cancer patients. Targeting this pathway might represent a promising treatment option. To this end, we investigated the anti-tumor efficacy of the dual PI3K/mTOR-inhibitor GDC-0084, which has been shown to cross the BBB and to achieve good metabolic stability in the brain (25). A phase-I trial on GDC-0084 in patients with progressive or recurrent high-grade glioma was recently completed (NCT01547546) with FDG-PET data showing that GDC-0084 is able to cross the BBB in humans and achieves a homogeneous distribution in the brain. Furthermore, $18.5 \%$ (5/27) of evaluable patients showed a metabolic partial response (37).

Phase-II trials are currently evaluating the efficacy of the PI3K-inhibitor buparlisib in patients with BCBM (NCT02000882) and in melanoma brain metastases (NCT02452294). The mTOR-inhibitor everolimus was recently assessed in combination with vinorelbine and trastuzumab in the treatment of HER2-positive, progressive breast cancer brain metastases (NCT01305941) and yielded low intracranial response rates (4\%) (38). Similarly, preliminary results of a recent phase Ib/II single-arm trial (NCT01783756) showed that the combination of everolimus, lapatinib and capecitabine for the treatment of HER2-positive breast cancer with brain metastases resulted in a $27 \%$ response rate in the brain after 12 weeks of treatment, highlighting the need for better therapies in this setting (39).

However, recent preclinical studies indicate that more favorable response rates can be achieved with dual inhibition of PI3K and mTOR (40), which further emphasizes the potential therapeutic advantages of GDC-0084. GDC-0084 has not been investigated in brain metastases patients to date.

We demonstrated in vitro that GDC-0084 induced apoptosis selectively in PIK3CA-MT BCBM cell lines at different magnitudes that corresponded to the extent of inhibition of molecular downstream targets of mTOR. These results were further validated with an isogenic epithelial breast cell line harboring a PIK3CA mutation. These findings are in line with a recent study in $\mathrm{CSCC}$, which demonstrated that GDC-0084 dose-dependently decreased cell viability, increased caspase 3 and caspase 9 activity and the proportion of cells in the G0/1 phase, and decreased the proportion of cells in the S- and G2M-phase (26).

In a patient-derived brain metastasis mouse model, we demonstrated that $15 \mathrm{mg} / \mathrm{kg} / \mathrm{day}$ of GDC-0084 administered orally significantly inhibited tumor growth in a trastuzumabresistant, PIK3CA-MT cell line model and not a PIK3CA-WT cell line model. PI3Kpathway inhibition within the brain was confirmed with immunohistochemistry by reduction in signal intensity for pAkt and pS6 ribosomal protein in the PIK3CA-MT tumors treated with GDC-0084. These results are in accordance with previous in vivo results of GDC-0084 in a glioblastoma (27) and in a cSCC (26) model, which showed that GDC-0084 significantly reduced tumor volumes and resulted in reduced phosphorylation of downstream targets of the PI3K pathway $(26,27)$. Of note, mice in the cSCC study received a higher dose of either 25 or $50 \mathrm{mg} / \mathrm{kg} /$ day of GDC-0084 (26).

We investigated whether inhibition of the PI3K/Akt/mTOR-pathway may cause a compensatory upregulation of the Ras/Raf/MAPK-signaling cascade, as it has been previously shown $(33,34)$ and we did not observe GDC-0084-induced changes in the 
Ras/Raf/MAPK- pathway signaling in both responsive and non-responsive breast cancer models.

In summary, our findings highlight that the effect of GDC-0084 is genotype-selective, leading to a significant response in treated PIK3CA-MT breast cancer cell lines compared to WT. These results are of substantial translational relevance, particularly with regard to BCBM patients with resistance to HER2-directed treatment approaches (41). Recent preclinical data suggest that activation of the PI3K/Akt/mTOR-pathway, including mechanisms like HER3 activation and PTEN loss is a frequent mediator of drug-resistance towards HER2-targeted agents in the brain $(41,42)$. Combined PI3K and mTOR-pathway blockade has been shown to overcome these resistance mechanisms (40) and may therefore present a promising treatment approach in affected patients in the future.

Our data therefore underscore the importance of brain-penetrant agents targeting the PI3K/Akt/mTOR-pathway. The experimental results from this study provide valuable preclinical support for the therapeutic efficacy of GDC-0084 in BCBM patients with dysregulated PI3K/mTOR signaling pathway. In future studies, in vitro and in vivo models in different genetic contexts will help identify additional biomarkers of response to PI3K inhibition. Although our preclinical results suggest a promising treatment option for affected patients with GDC-0084 as a single agent, there is an urgent need to explore combinatorial targeted treatment approaches to further improve intracranial tumor control and consequently, overall survival in breast BCBM patients. These findings warrant further validation in clinical trials in order to explore the therapeutic benefit of GDC-0084 in patients. A national multi-center trial is planned to evaluate the efficacy of GDC-0084 in brain metastases harboring PIK3CA mutations (Alliance study A071701).

\section{Supplementary Material}

Refer to Web version on PubMed Central for supplementary material.

\section{References}

1. Asher AL, Burri SH, Chahlavi A, Chang SM, Farace E, Fiveash JB, et al. The Management of brain metastases In: Schiff D, O’Neill BP, editors. Principles of Neuro-Oncology. 1st Edition ed. New York: McGraw-Hill Medical Publishing Division; 2005 p. 553-79.

2. Eichler AF, Loeffler JS. Multidisciplinary management of brain metastases. The oncologist. 2007;12(7):884-98. [PubMed: 17673619]

3. Soffietti R, Ducati A, Rudà R. Brain metastases In: Aminoff MJ, Boller F, Swaab DF, editors. Handbook of Clinical Neurology. 105: Elsevier; 2012 p. 747-55.

4. Kocher M, Soffietti R, Abacioglu U, Villa S, Fauchon F, Baumert BG, et al. Adjuvant whole-brain radiotherapy versus observation after radiosurgery or surgical resection of one to three cerebral metastases: results of the EORTC 22952-26001 study. Journal of clinical oncology : official journal of the American Society of Clinical Oncology. 2011;29(2):134-41. [PubMed: 21041710]

5. Murray KJ, Scott C, Greenberg HM, Emami B, Seider M, Vora NL, et al. A randomized phase III study of accelerated hyperfractionation versus standard in patients with unresected brain metastases: a report of the Radiation Therapy Oncology Group (RTOG) 9104. International journal of radiation oncology, biology, physics. 1997;39(3):571-4.

6. Shah R, Rosso K, Nathanson SD. Pathogenesis, prevention, diagnosis and treatment of breast cancer. World Journal of Clinical Oncology. 2014;5(3):283-98. [PubMed: 25114845] 
7. Witzel I, Oliveira-Ferrer L, Pantel K, Müller V, Wikman H. Breast cancer brain metastases: biology and new clinical perspectives. Breast cancer research : BCR. 2016;18:8. [PubMed: 26781299]

8. Adamo B, Deal AM, Burrows E, Geradts J, Hamilton E, Blackwell KL, et al. Phosphatidylinositol 3-kinase pathway activation in breast cancer brain metastases. Breast cancer research : BCR. 2011;13(6):R125. [PubMed: 22132754]

9. Brastianos PK, Carter SL, Santagata S, Cahill DP, Taylor-Weiner A, Jones RT, et al. Genomic Characterization of Brain Metastases Reveals Branched Evolution and Potential Therapeutic Targets. Cancer discovery. 2015;5(11):1164-77. [PubMed: 26410082]

10. Saunus JM, Quinn MC, Patch AM, Pearson JV, Bailey PJ, Nones K, et al. Integrated genomic and transcriptomic analysis of human brain metastases identifies alterations of potential clinical significance. The Journal of pathology. 2015;237(3):363-78. [PubMed: 26172396]

11. Sadeghi N, Gerber DE. Targeting the PI3K pathway for cancer therapy. Future medicinal chemistry. 2012;4(9):1153-69. [PubMed: 22709255]

12. de Gooijer MC, Zhang P, Buil LCM, Çitirikkaya CH, Thota N, Beijnen JH, et al. Buparlisib is a brain penetrable pan-PI3K inhibitor. Scientific reports. 2018;8(1):10784. [PubMed: 30018387]

13. Lin NU. Breast cancer brain metastases: new directions in systemic therapy. Ecancermedicalscience. 2013;7:307. [PubMed: 23662165]

14. O’Reilly T, McSheehy PM, Kawai R, Kretz O, McMahon L, Brueggen J, et al. Comparative pharmacokinetics of RAD001 (everolimus) in normal and tumor-bearing rodents. Cancer chemotherapy and pharmacology. 2010;65(4):625-39. [PubMed: 19784839]

15. Brana I, LoRusso P, Baselga J, Heath EI, Patnaik A, Gendreau S, et al. A phase I dose-escalation study of the safety, pharmacokinetics (PK), and pharmacodynamics of XL765 (SAR245409), a $\mathrm{PI} 3 \mathrm{~K} / \mathrm{TORC} 1 / \mathrm{TORC} 2$ inhibitor administered orally to patients (pts) with advanced malignancies. 2010 ASCO Annual Meeting2010.

16. Cloughesy TF, Mischel PS, Omuro AMP, Prados M, Wen PY, Wu B, et al. Tumor pharmacokinetics (PK) and pharmacodynamics (PD) of SAR245409 (XL765) and SAR245408 (XL147) administered as single agents to patients with recurrent glioblastoma (GBM): An Ivy Foundation early-phase clinical trials consortium study. 2013 ASCO Annual Meeting 2013.

17. Franz DN, Belousova E, Sparagana S, Bebin EM, Frost M, Kuperman R, et al. Efficacy and safety of everolimus for subependymal giant cell astrocytomas associated with tuberous sclerosis complex (EXIST-1): a multicentre, randomised, placebo-controlled phase 3 trial. Lancet. 2013;381(9861):125-32. [PubMed: 23158522]

18. Koul D, Shen R, Kim YW, Kondo Y, Lu Y, Bankson J, et al. Cellular and in vivo activity of a novel PI3K inhibitor, PX-866, against human glioblastoma. Neuro-oncology. 2010;12(6):559-69. [PubMed: 20156803]

19. Liu TJ, Koul D, LaFortune T, Tiao N, Shen RJ, Maira SM, et al. NVP-BEZ235, a novel dual phosphatidylinositol 3-kinase/mammalian target of rapamycin inhibitor, elicits multifaceted antitumor activities in human gliomas. Molecular cancer therapeutics. 2009;8(8):2204-10. [PubMed: 19671762]

20. Paplomata E, O'Regan R. The PI3K/AKT/mTOR pathway in breast cancer: targets, trials and biomarkers. Therapeutic advances in medical oncology. 2014;6(4):154-66. [PubMed: 25057302]

21. Niessner H, Schmitz J, Tabatabai G, Schmid AM, Calaminus C, Sinnberg T, et al. PI3K Pathway Inhibition Achieves Potent Antitumor Activity in Melanoma Brain Metastases In Vitro and In Vivo. Clinical cancer research : an official journal of the American Association for Cancer Research. 2016;22(23):5818-28.

22. Osswald M, Blaes J, Liao Y, Solecki G, Gommel M, Berghoff AS, et al. Impact of Blood-Brain Barrier Integrity on Tumor Growth and Therapy Response in Brain Metastases. Clinical cancer research : an official journal of the American Association for Cancer Research. 2016;22(24):607887. [PubMed: 27521448]

23. Chen G, Chakravarti N, Aardalen K, Lazar AJ, Tetzlaff MT, Wubbenhorst B, et al. Molecular profiling of patient-matched brain and extracranial melanoma metastases implicates the PI3K pathway as a therapeutic target. Clinical cancer research : an official journal of the American Association for Cancer Research. 2014;20(21):5537-46. 
24. Becker CM, Oberoi RK, McFarren SJ, Muldoon DM, Pafundi DH, Pokorny JL, et al. Decreased affinity for efflux transporters increases brain penetrance and molecular targeting of a PI3K/mTOR inhibitor in a mouse model of glioblastoma. Neuro-oncology. 2015;17(9):1210-9. [PubMed: 25972455]

25. Heffron TP, Ndubaku CO, Salphati L, Alicke B, Cheong J, Drobnick J, et al. Discovery of Clinical Development Candidate GDC-0084, a Brain Penetrant Inhibitor of PI3K and mTOR. ACS Medicinal Chemistry Letters. 2016.

26. Ding LT, Zhao P, Yang ML, Lv GZ, Zhao TL. GDC-0084 inhibits cutaneous squamous cell carcinoma cell growth. Biochemical and biophysical research communications. 2018.

27. Salphati L, Alicke B, Heffron TP, Shahidi-Latham S, Nishimura M, Cao T, et al. Brain Distribution and Efficacy of the Brain Penetrant PI3K Inhibitor GDC-0084 in Orthotopic Mouse Models of Human Glioblastoma. Drug Metab Dispos. 2016;44(12):1881-9. [PubMed: 27638506]

28. Jernström S, Hongisto V, Leivonen S-K, Due EU, Tadele DS, Edgren H, et al. Drug-screening and genomic analyses of HER2-positive breast cancer cell lines reveal predictors for treatment response. Breast cancer (Dove Medical Press). 2017;9:185-98. [PubMed: 28356768]

29. Kataoka Y, Mukohara T, Shimada H, Saijo N, Hirai M, Minami H. Association between gain-offunction mutations in PIK3CA and resistance to HER2-targeted agents in HER2-amplified breast cancer cell lines. Annals of Oncology. 2010;21(2):255-62. [PubMed: 19633047]

30. Gustin JP, Karakas B, Weiss MB, Abukhdeir AM, Lauring J, Garay JP, et al. Knockin of mutant PIK3CA activates multiple oncogenic pathways. Proceedings of the National Academy of Sciences of the United States of America. 2009;106(8):2835-40. [PubMed: 19196980]

31. Iqbal A, Eckerdt F, Bell J, Nakano I, Giles FJ, Cheng SY, et al. Targeting of glioblastoma cell lines and glioma stem cells by combined PIM kinase and PI3K-p110alpha inhibition. Oncotarget. 2016;7(22):33192-201. [PubMed: 27120806]

32. Khalil AA, Jameson MJ, Broaddus WC, Lin PS, Dever SM, Golding SE, et al. The Influence of Hypoxia and $\mathrm{pH}$ on Bioluminescence Imaging of Luciferase-Transfected Tumor Cells and Xenografts. Int J Mol Imaging. 2013;2013:287697. [PubMed: 23936647]

33. Butler DE, Marlein C, Walker HF, Frame FM, Mann VM, Simms MS, et al. Inhibition of the $\mathrm{PI} 3 \mathrm{~K} / \mathrm{AKT} / \mathrm{mTOR}$ pathway activates autophagy and compensatory Ras/Raf/MEK/ERK signalling in prostate cancer. Oncotarget. 2017;8(34):56698-713. [PubMed: 28915623]

34. Yuen HF, Abramczyk O, Montgomery G, Chan KK, Huang YH, Sasazuki T, et al. Impact of oncogenic driver mutations on feedback between the PI3K and MEK pathways in cancer cells. Biosci Rep. 2012;32(4):413-22. [PubMed: 22668349]

35. O'Brien NA, Browne BC, Chow L, Wang Y, Ginther C, Arboleda J, et al. Activated phosphoinositide 3-kinase/AKT signaling confers resistance to trastuzumab but not lapatinib. Molecular cancer therapeutics. 2010;9(6):1489-502. [PubMed: 20501798]

36. Leyland-Jones B Human epidermal growth factor receptor 2-positive breast cancer and central nervous system metastases. Journal of clinical oncology : official journal of the American Society of Clinical Oncology. 2009;27(31):5278-86. [PubMed: 19770385]

37. Wen PY, Cloughesy TF, Olivero A, Lu X, Mueller L, Coimbra AF, et al. A first-in-human phase 1 study to evaluate the brain-penetrant PI3K/mTOR inhibitor GDC-0084 in patients with progressive or recurrent high-grade glioma. Journal of Clinical Oncology. 2016;34(15_suppl):2012-.

38. Van Swearingen AED, Siegel MB, Deal AM, Sambade MJ, Hoyle A, Hayes DN, et al. LCCC 1025: a phase II study of everolimus, trastuzumab, and vinorelbine to treat progressive HER2positive breast cancer brain metastases. Breast cancer research and treatment. 2018.

39. Hurvitz S, Singh R, Adams B, Taguchi JA, Chan D, Dichmann RA, et al. Phase Ib/II single-arm trial evaluating the combination of everolimus, lapatinib and capecitabine for the treatment of HER2-positive breast cancer with brain metastases (TRIO-US B-09). Therapeutic advances in medical oncology. 2018;10:1758835918807339.

40. Ni J, Ramkissoon SH, Xie S, Goel S, Stover DG, Guo H, et al. Combination inhibition of PI3K and mTORC1 yields durable remissions in mice bearing orthotopic patient-derived xenografts of HER2-positive breast cancer brain metastases. Nature medicine. 2016;22(7):723-6.

41. Kabraji S, Ni J, Lin NU, Xie S, Winer EP, Zhao JJ. Drug Resistance in HER2-Positive Breast Cancer Brain Metastases: Blame the Barrier or the Brain? Clinical cancer research : an official 
journal of the American Association for Cancer Research. 2018;24(8):1795-804. [PubMed: 29437794]

42. Kodack DP, Askoxylakis V, Ferraro GB, Sheng Q, Badeaux M, Goel S, et al. The brain microenvironment mediates resistance in luminal breast cancer to PI3K inhibition through HER3 activation. Science translational medicine. 2017;9(391). 


\section{Translational relevance:}

Although substantive efforts have been undertaken to optimize multidisciplinary treatment approaches for patients with breast cancer brain metastases, patients still have a dismal overall survival. In recent years, genomic studies have tremendously improved our understanding of actionable molecular targets in patients with breast cancer brain metastases. The PI3K/Akt/mTOR-pathway has been shown to be upregulated in a majority of affected patients. However, there are still no approved systemic agents targeting this pathway to date, and drug administration in the central nervous system is complicated by the inherent properties of the blood-brain barrier. In this study, we demonstrate that the brain-penetrant dual PI3K/mTOR-inhibitor GDC-0084 significantly inhibits tumor growth in a preclinical model of PIK3CA-mutant breast cancer brain metastasis. The results of our study therefore suggest that GDC-0084 might be a promising treatment strategy for patients with $P I K 3 C A$-mutant breast cancer brain metastases and warrant further validation in clinical trials. 
A B

JIMT-1 BR-3

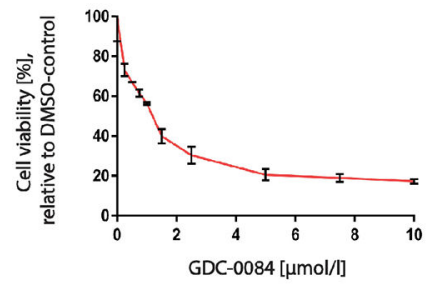

MDA-MB-361

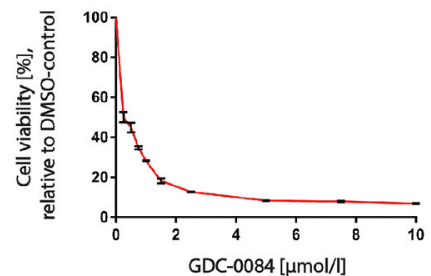

MDA-MB-231 BrM2

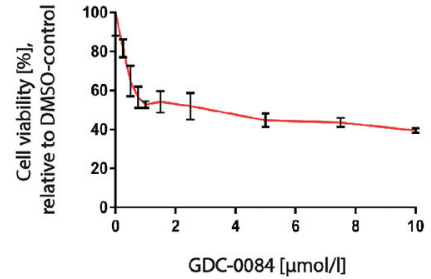

BS-004

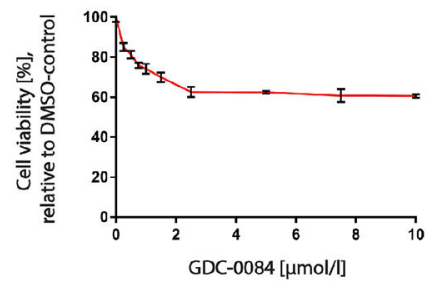

JIMT-1 BR-3

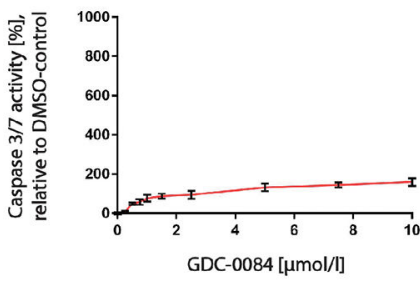

MDA-MB-361

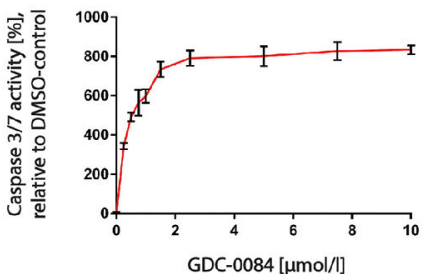

MDA-MB-231 BrM2

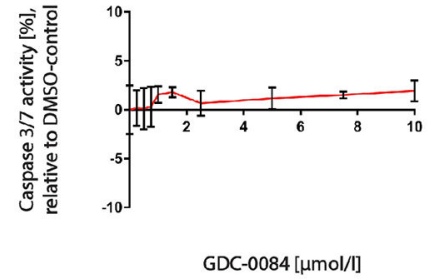

BS-004

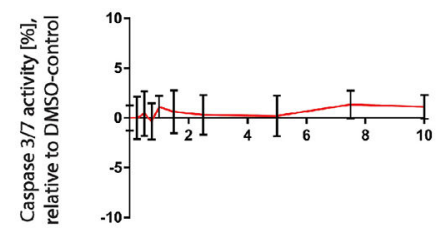

GDC-0084 [ $\mu \mathrm{mol} / 1]$
JIMT-1 BR-3

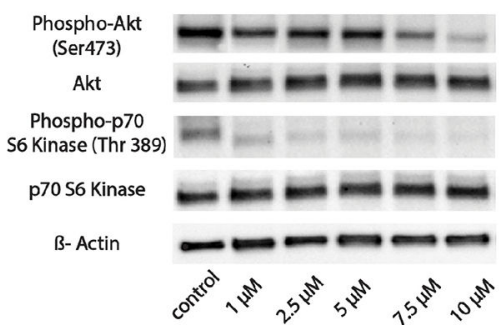

MDA-MB-361

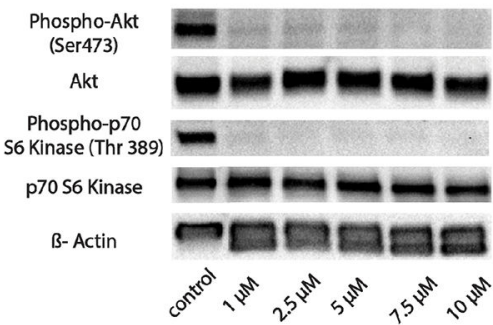

MDA-MB-231 BrM2

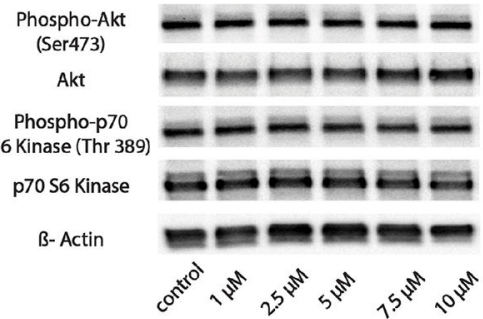

BS-004

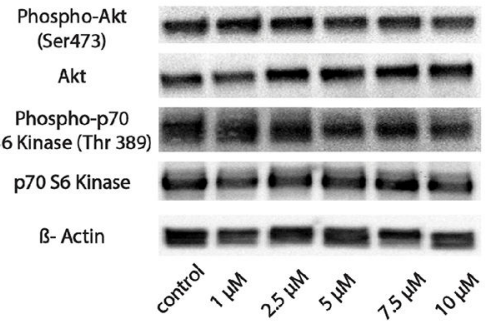

Figure 1.

The dual PI3K/mTOR-inhibitor GDC-0084 selectively reduces cell viability and induces apoptosis in the PIK3CA-mutant cell lines JIMT-1 BR-3 and MDA-MB-361 compared to the PIK3CA-wildtype cell lines MDA-MB-231 BrM2 and BS-004

A, CellTiter Glo cell viability assays after 72 hours of treatment and B, Caspase 3/7 Glo apoptosis assays after 10 hours of treatment of the breast cancer brain metastases cell lines JIMT-1 BR-3, MDA-MB-361, MDA-MB-231 BrM2 and BS-004 in increasing concentrations with the dual PI3K/mTOR-inhibitor GDC-0084. Percentage of growth 
inhibiton is compared to 0.1\% DMSO-incubated controls. Error bars represent SEM of cells seeded in triplicates and treated with the same dose. $\mathbf{C}$, Western blot analysis of pAkt (Ser473), Akt, p-p70 S6 Kinase (Thr389) and p70 S6 Kinase in JIMT-1 BR-3, MDAMB-361, MDA-MB-231 BrM2 and BS-004 cell lines treated with GDC-0084 in increasing concentrations for a total of 6 hours. $\beta$-Actin was used as a loading control. Western blots are representative of three independent experiments per cell line. 


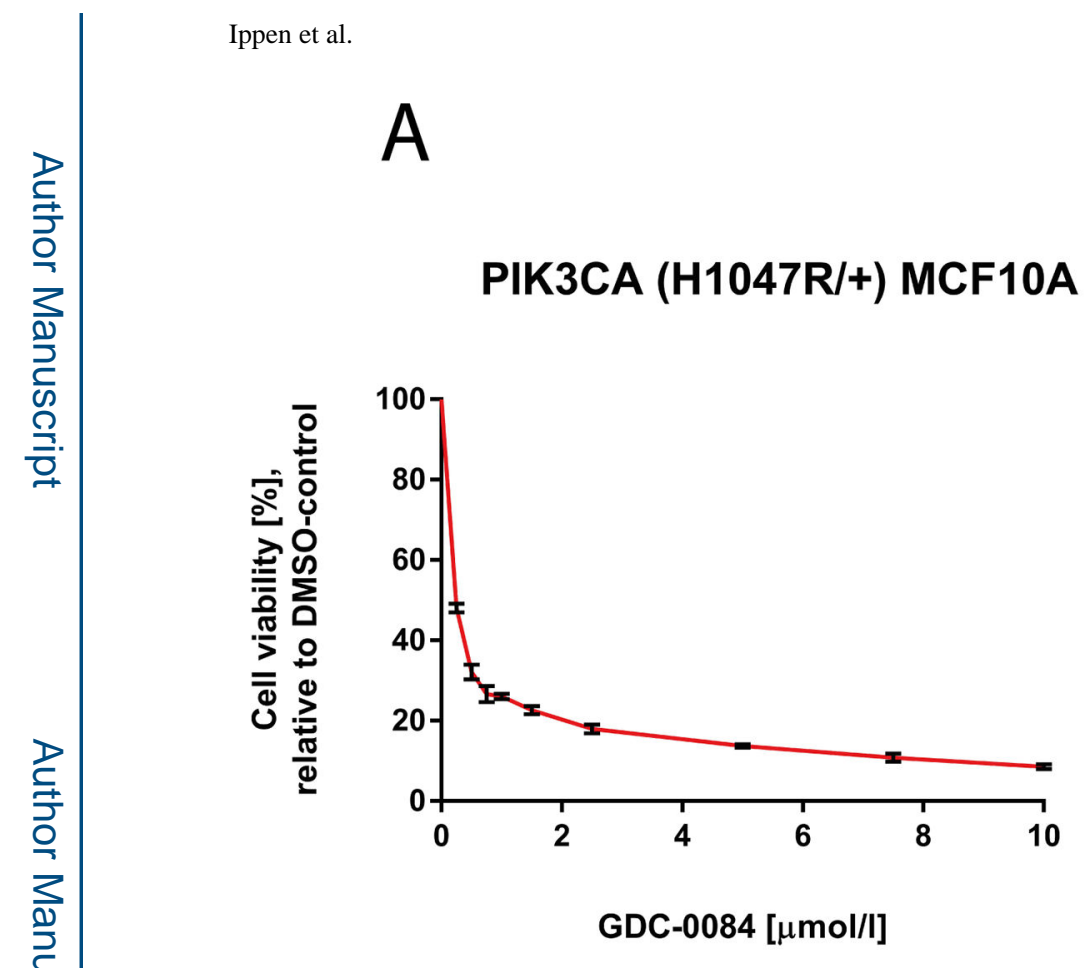

MCF10A (parental)

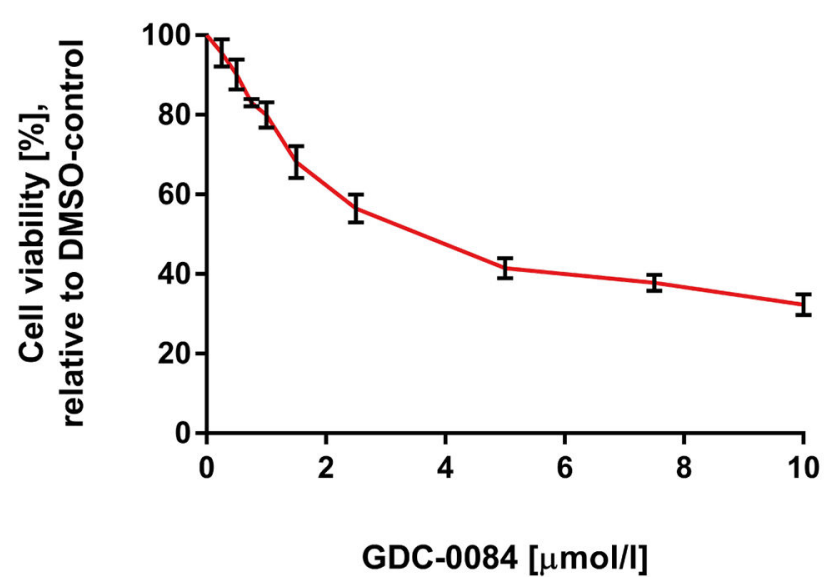

B

PIK3CA (H1047R/+) MCF10A

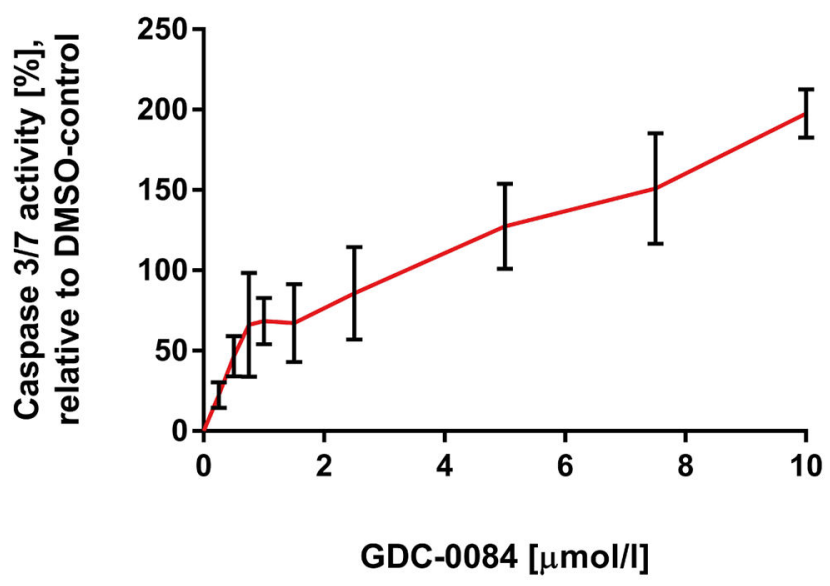

MCF10A (parental)

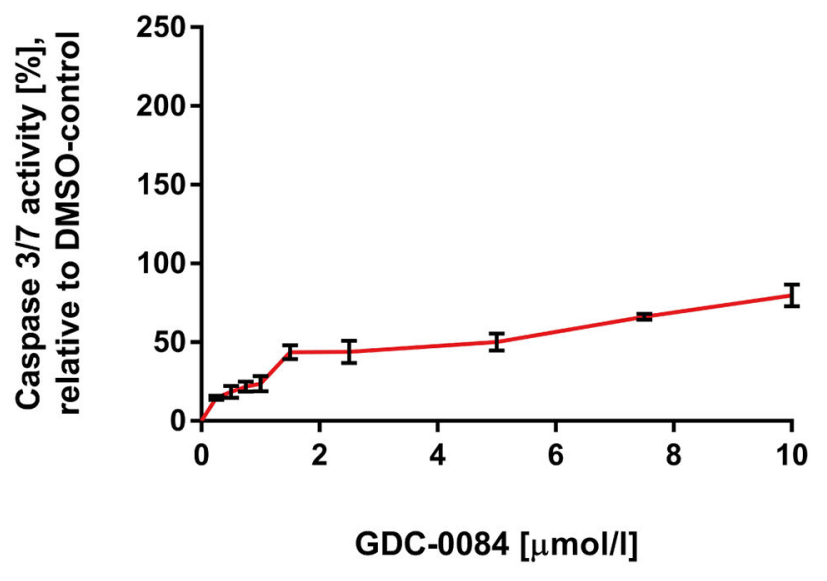

Figure 2.

GDC-0084 significantly decreases cell viability and induces apoptosis in the PIK3CAmutant epithelial breast cell line PIK3CA (H1047R/+) MCF10A compared to the isogenic $P I K 3 C A$-wildtype parental epithelial breast cell line MCF10A.

A, CellTiter Glo cell viability assays after 72 hours of treatment and B, Caspase 3/7 Glo apoptosis assays after 24 hours of treatment of the epithelial breast cell lines PIK $3 C A$ (H1047R/+) MCF10A and MCF10A in increasing concentrations with the dual PI3K/ mTOR-inhibitor GDC-0084. Percentage of growth inhibiton is compared to $0.1 \%$ DMSOincubated controls. Error bars represent SEM of cells seeded in triplicates and treated with the same dose. 
A

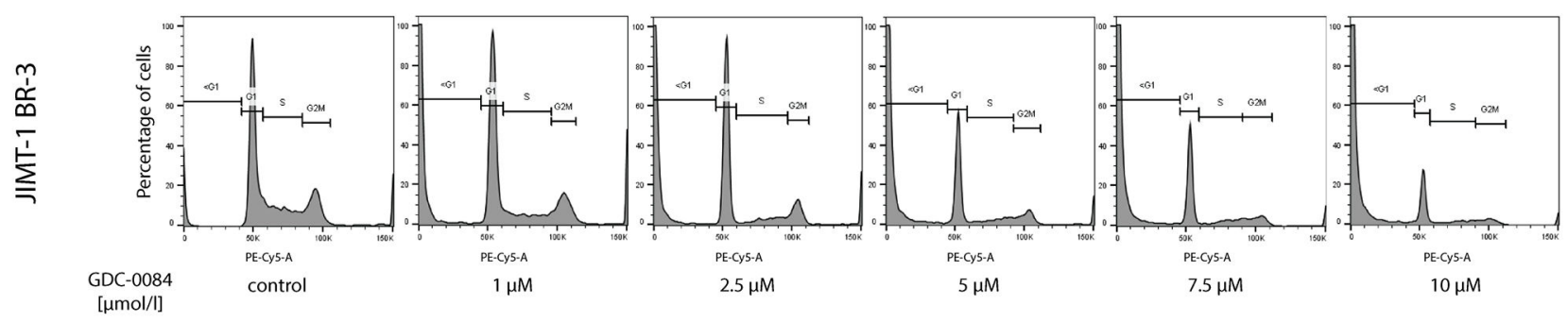

B

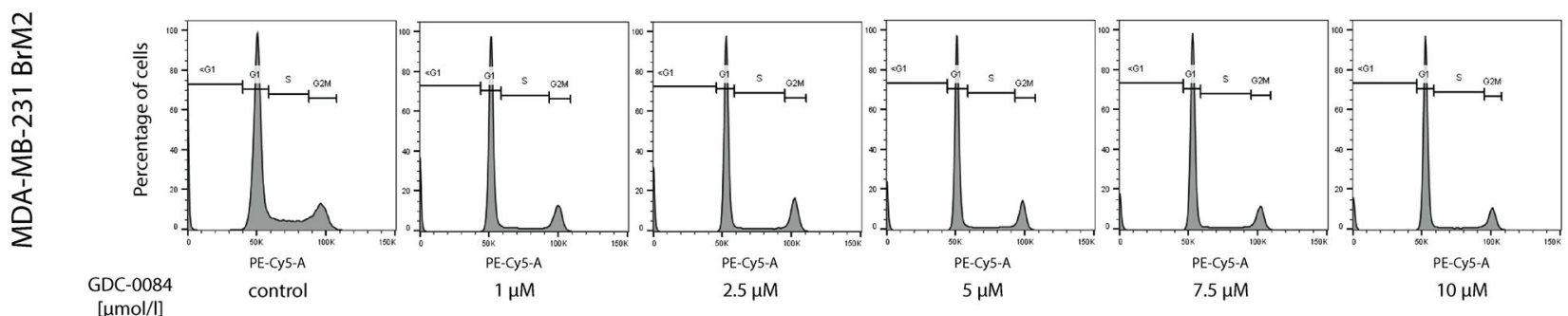

JIMT-1 BR-3

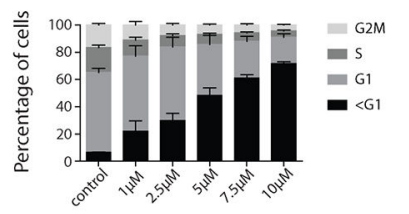

GDC-0084 [umol/l]
MDA-MB-361

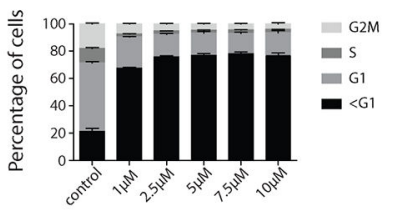

GDC-0084 [umo/l]
MDA-MB-231 BrM2

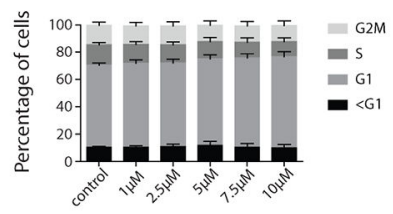

GDC-0084 [umol/l]
BS-004

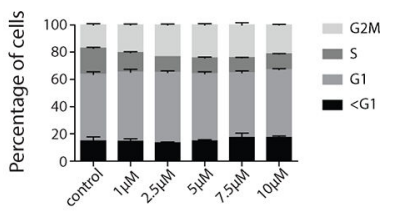

GDC-0084 [umol/l]

Figure 3.

GDC-0084 induces apoptosis in the PIK3CA-mutant cell lines JIMT-1 BR-3 and MDA-

MB-361 and growth inhibition in the PIK3CA-wildtype cell lines MDA-MB-231 BrM2 and

BS-004. The four breast cancer brain metastases cell lines were treated in increasing concentrations with GDC-0084 for 72 hours, stained with propidium iodide (PI) and underwent subsequent cell cycle analysis on the LSRII.

The cell cycle for A, JIMT-1 BR-3 and B MDA-MB-231 BrM2, treated in increasing concentrations of the inhibitor is displayed as a histogram. Each phase of the cell cycle $(<\mathrm{G} 1, \mathrm{G} 1, \mathrm{~S}, \mathrm{G} 2 \mathrm{M})$ is gated on the histogram. C, Dose-dependent percentages of phases of the cell cycle were assessed in three independent experiments for each cell line (JIMT-1 BR-3, MDA-MB-361, MDA-MB-231 BrM2 and BS-004) and displayed in a vertical bar graph. All calculations are relative to $0.1 \%$ DMSO-incubated controls. Error bars represent SEM of each phase of the cell cycle. See Supplementary Fig. 2 for cell cycle analysis for MDA-MB-361 and BS-004. 

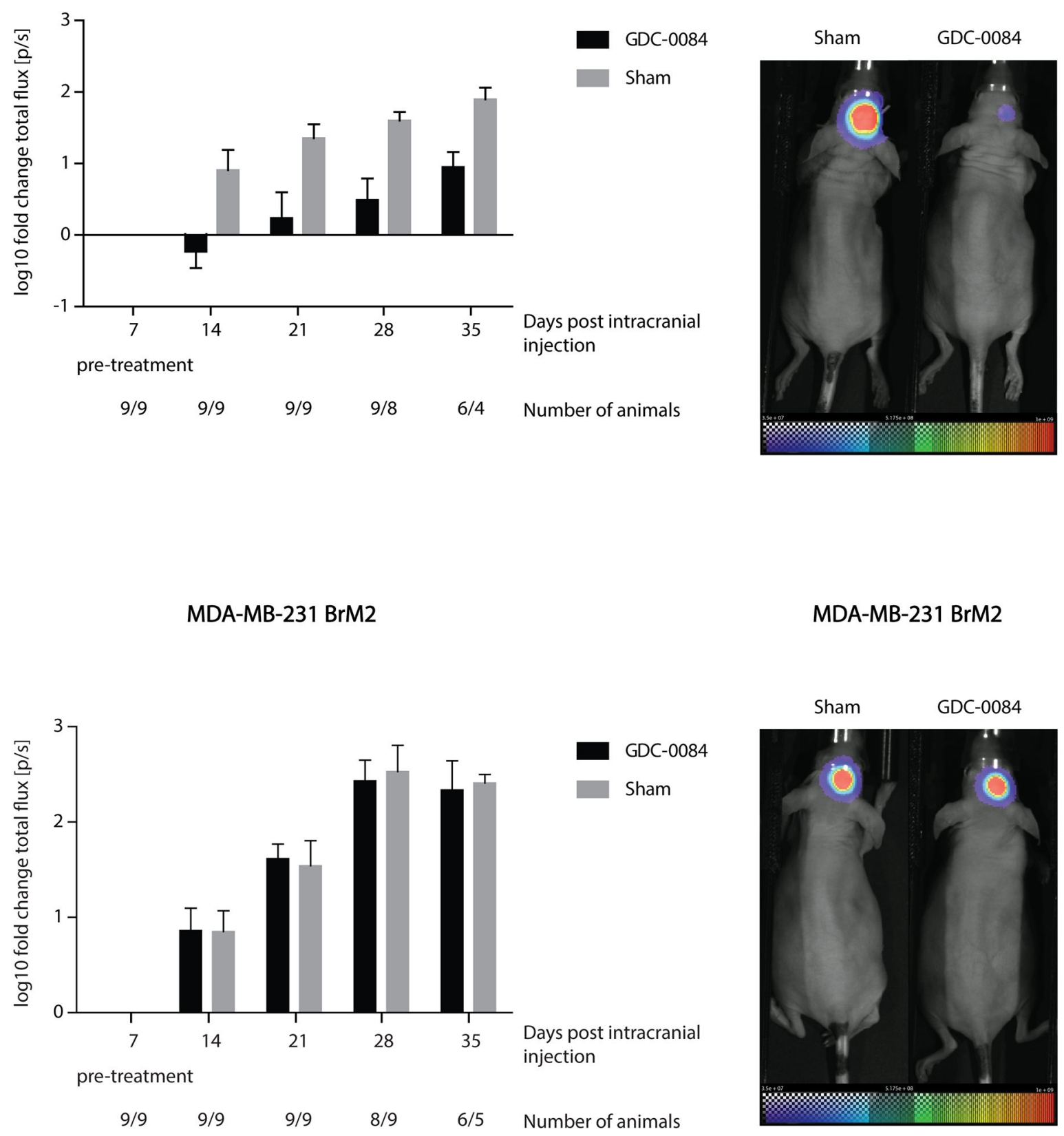

Figure 4.

GDC-0084 inhibits tumor growth in the brain in vivo. Tumor growth was analyzed via bioluminescent imaging (BLI) and quantified as log10-transformed fold-change of total flux [p/s]. Imaging was performed 7 days after intracranial injection of tumor cells (pretreatment) and every 7 days thereafter until the $35^{\text {th }}$ day post-injection. GDC-0084 significantly inhibited tumor growth compared to sham-treatment in the mouse model using A, the PIK3CA-mutant cell line JIMT-1 BR-3 (A). No benefit was detected in the treatmentcohort compared to sham controls in B, the PIK3CA-wildtype MDA-MB-231 BrM2 model. 
Error bars represent SEM of log10-transformed total flux fold change values. Representative BLI-images for treated and control mice of each model at the last day of imaging (A, JIMT-1 BR-3 and B, MDA-MB-231 BrM2) are shown on right. See Supplementary Fig. 3 for changes in the flux for the individual animals over time. 
JIMT-1 BR-3

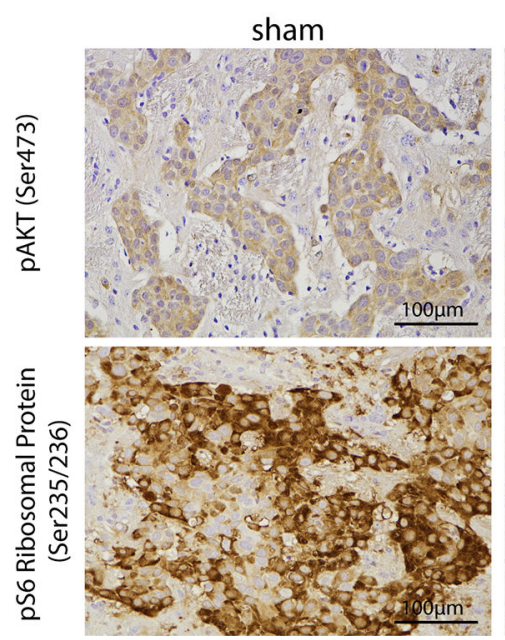

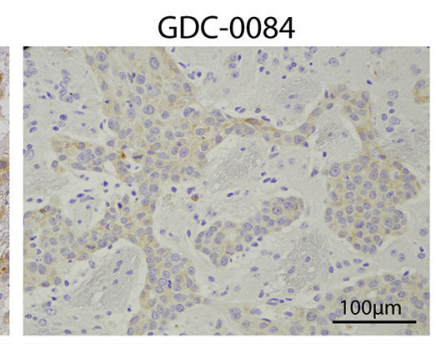

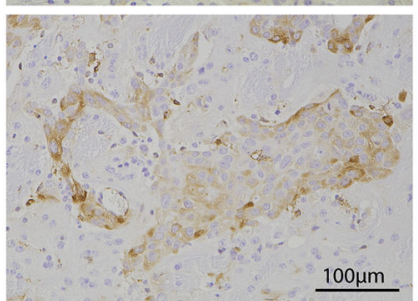

MDA-MB-231 BrM2
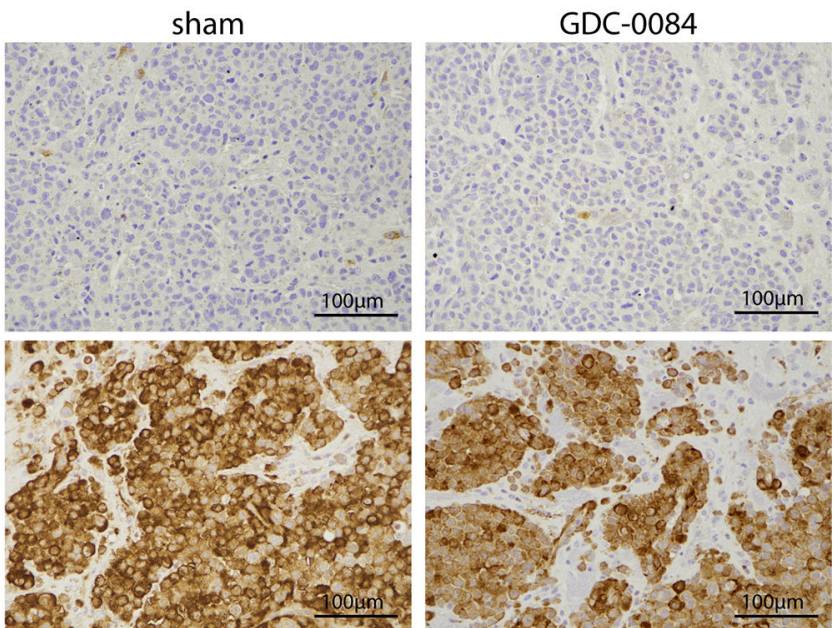

Figure 5.

Photomicrographs illustrating pAkt (Ser473) and pS6 ribosomal protein (Ser235/236) expression in mouse brains harboring either JIMT-1 BR-3 or MDA-MB-231 BrM2 intracranial tumors, stratified by treatment with sham or GDC-0084. Staining for pAkt and pS6 ribosomal protein was considerably weaker in PIK3CA-mutant (JIMT-1 BR-3) tumors treated with GDC-0084 compared to sham. No clear differences in staining intensity were found in PIK3CA-wildtype tumors (MDA-MB-231 BrM2) regardless of treatment. 